\title{
Maximum directed cuts in graphs with degree constraints
}

\author{
Baogang $\mathrm{Xu}^{*}$ \\ School of Mathematics \& Computer Science \\ Nanjing Normal University \\ 122 Ninghai Road, Nanjing, 210097, China \\ Email: baogxu@njnu.edu.cn \\ Xingxing $\mathrm{Yu}^{\dagger}$ \\ School of Mathematics \\ Georgia Institute of Technology \\ Atlanta, GA 30332-0160, USA \\ Email:yu@math.gatech.edu
}

\begin{abstract}
The Max Cut problem is an NP-hard problem and has been studied extensively. Alon et al. studied a directed version of the Max Cut problem and observed its connection to the Hall ratio of graphs. They proved, among others, that if an acyclic digraph has $m$ edges and each vertex has indegree or outdegree at most 1 , then it has a directed cut of size at least $2 m / 5$. Lehel et al. extended this result to all digraphs without directed triangles. In this paper, we characterize the acyclic digraphs with $m$ edges whose maximum dicuts have exactly $2 m / 5$ edges, and our approach gives an alternative proof of the result of Lehel et al. We also show that there are infinitely many positive rational numbers $\beta<2 / 5$ for which there exist digraphs $D$ (with directed triangles) such that each vertex of $D$ has indegree or outdegree at most 1 , and any maximum directed cut in $D$ has size precisely $\beta|E(D)|$.
\end{abstract}

Key words and phrases: maximum directed cut, digraph, subcubic graph, triangle-free graph AMS 2000 Subject Classifications: 05C35,05C75

\footnotetext{
*Partially supported by NSFC Project 10671095, and Georgia Institute of Technology.

${ }^{\dagger}$ Partially supported by NSA and NSFC Project 10628102
} 


\section{Introduction}

The Max Cut Problem has been studied extensively from both combinatorial and computational perspectives. This problem is one of the Karp's original NP-complete problems [12], and remains NP-hard even when restricted to triangle-free cubic graphs [17]. It is shown in [3] that it is NPhard to approximate the Max Cut problem on cubic graphs beyond the ratio of 0.997. Goemans and Williamson [9] used semidefinite programming to give a randomized algorithm with expected performance guarantee of 0.87856. Feige, Karpinski, and Langberg [8] gave a similar randomized algorithm that improves this bound to .921 for subcubic graphs. A graph is subcubic if it has maximum degree at most three.

Edwards [7] proved that every graph of size $m$ has a cut of size at least $m / 2+\sqrt{m / 8+1 / 64}-$ $1 / 8$, and this lower bound is attained by complete graphs of odd order. Alon [1] showed that Edwards' bound is essentially best possible in the sense that there is a constant $c>0$ such that for every integer $m>0$, there exists a graph of size $m$ in which any maximum cut has size at most $m / 2+\sqrt{m / 8}+\mathrm{cm}^{1 / 4}$. On the other hand, Edwards' bound has been improved substantially for subcubic graphs. See, for example, $[4,10,14-16]$. Some of these results on subcubic graphs will be used later in this paper.

A digraph version of the Max Cut problem is to find, in a digraph, a directed cut (dicut for short) with maximum number of edges. Let $D$ be a digraph, and let $S \subseteq V(D)$. We use $(S, \bar{S})$ to denote the dicut which consists of all edges in $D$ that are directed from $S$ to $\bar{S}:=V-S$. It is easy to see from Edwards' bound above that every digraph of size $m$ has a dicut of size at least $m / 4+\sqrt{m / 32+1 / 256}-1 / 16$, and the regular orientations of complete graphs of odd order show that this bound is tight.

This lower bound can be improved for acyclic digraphs. Alon et al. [2] observed that maximum dicuts of acyclic digraphs are related to the Hall ratio of graphs $[5,6,11]$, and proved that every acyclic digraph of size $m$ has a dicut of size $m / 4+\Omega\left(m^{4 / 5}\right)$. They also showed that this bound can be improved further by adding restrictions on vertex degrees. For positive integers $k$ and $l$, let $D(k, l)$ denote the family of digraphs such that every vertex has indegree at most $k$ or outdegree at most $l$. It is proved in [2] that every digraph in $D(k, l)$ of size $m$ has a dicut of size at least $(k+l+2) m /(4 k+4 l+6)$.

In this paper, we are primarily concerned with the class $D(1,1)$, which includes those digraphs whose underlying graphs are subcubic. Let $D$ be a digraph. We use $\varepsilon(D)$ to denote the number of edges in $D$, and define the dicut density of $D$ as

$$
\gamma(D):=\max \{|(S, \bar{S})| / \varepsilon(D): S \subseteq V(D)\} .
$$

Alon et al. [2] showed that if $D \in D(1,1)$ and $D$ is acyclic then $\gamma(D) \geq 2 / 5$; and the bound is attained by the digraph $A$ which consists of the directed path $x_{0} x_{1} x_{2} x_{3} x_{4}$ and a directed edge from $x_{1}$ to $x_{3}$ (see Figure 5). In fact, the digraph $A$ and the directed pentagon are the only two digraphs that have at most 5 edges, contain no directed triangles, and have dicut density exactly 2/5. Recently, Lehel et al. [13] showed that if $D \in D(1,1)$ and $D$ contains no directed triangles then $\gamma(D) \geq 2 / 5$

One result in this paper is a characterization of all acyclic digraphs in $D(1,1)$ with dicut density $2 / 5$. (This problem was suggested to us by Lehel.) Our approach also gives an alternative proof of the result of Lehel et al. Moreover, for each integer $i \geq 0$ we find a digraph in $D(1,1)$ with dicut density $(1+2 i) /(3+5 i)<2 / 5$. So there are infinitely many rational numbers in the interval $[1 / 3,2 / 5]$ which could serve as the dicut density of a digraph in $D(1,1)$. 
Let $D$ be a digraph. When the underlying graph of $D$ is subcubic, we say that $D$ is subcubic. We use $D^{r}$ to denote the digraph obtained from $D$ by reversing the orientation of every edge in $D$. It is easy to see that $\gamma(D)=\gamma\left(D^{r}\right)$. We use $(x, y)$ to denote a directed edge from the vertex $x$ to the vertex $y$. Let $u$ be a vertex of $D$. Then $d_{D}^{+}(u)$ denotes the outdegree of $u$, and $d_{D}^{-}(u)$ denotes the indegree of $u$. The vertex $u$ is said to be a $k$-vertex in $D$ if $d_{D}^{+}(u)+d_{D}^{-}(u)=k$. For convenience, we define

$$
\mathcal{D}=\{D \in D(1,1): D \text { is connected and contains no directed triangle }\} .
$$

Note that all connected acyclic digraphs in $D(1,1)$ belong to $\mathcal{D}$.

In section 2 , we prove two structural lemmas. We then give an alternative proof of the result of Lehel et al. [13] by performing a simple operation on digraphs and applying a result of Bondy and Locke [4]. We also show that there are infinitely many rational numbers in the interval $[1 / 3,2 / 5]$ which could serve as the dicut density of a digraph in $D(1,1)$. In section 3 , we use the structural lemmas to study those digraphs with dicut density $2 / 5$. We show that when the operation introduced in section 2 is applied to an extremal graph, the resulting graph is also extremal. We then show that there are only two extremal graphs which are irreducible (and neither is acyclic), which enables us to characterize all extremal graphs that are acyclic.

\section{Structural lemmas}

Alon et al. showed that acyclic digraphs in $\mathcal{D}$ have dicut density at least $2 / 5$. The goal of this section is to prove two lemmas that will be used to characterize the extrmal graphs. As a byproduct, we also give an alternative proof of the result of Lehel et al. that all digraphs in $\mathcal{D}$ have dicut density at least $2 / 5$.

First, we define an operation on digraphs. Let $D$ be a digraph, $x \in V(D)$, and $\left(x, y_{i}\right) \in E(D)$ $(i=1,2)$, and assume that $d_{D}^{-}(x) \leq 1$ and $d_{D}^{+}\left(y_{i}\right) \leq 1$ for $i \in\{1,2\}$. Let $D^{\prime}$ be obtained from $D-\left\{\left(x, y_{1}\right),\left(x, y_{2}\right)\right\}$ by further deleting the possible edge directed towards $x$ and the possible edges directed away from $y_{1}$ or $y_{2}$. We say that $D$ is reducible (to $D^{\prime}$ ), and denote this by $D^{\prime}:=R\left(D, x, y_{1}, y_{2}\right)$. We say that $D$ is irreducible if neither $D$ nor $D^{r}$ is reducible.

Lemma 2.1 Let $D$ and $D^{\prime}:=R\left(D, x, y_{1}, y_{2}\right)$ be defined as above. Then

(a) $D^{\prime}$ has a maximum dicut $\left(S^{\prime}, \overline{S^{\prime}}\right)$ such that $x \in S^{\prime}$ and $\left\{y_{1}, y_{2}\right\} \subseteq \overline{S^{\prime}}$, and

(b) $\gamma\left(D^{\prime}\right) \geq 2 / 5$ implies $\gamma(D) \geq 2 / 5$.

Proof. Since $d_{D}^{-}(x) \leq 1$ and $d_{D}^{+}\left(y_{i}\right) \leq 1$ for $i \in\{1,2\}, \varepsilon\left(D^{\prime}\right) \geq \varepsilon(D)-5$ and $d_{D^{\prime}}^{-}(x)=d_{D^{\prime}}^{+}\left(y_{1}\right)=$ $d_{D^{\prime}}^{+}\left(y_{2}\right)=0$. Let $(S, \bar{S})$ be a maximum dicut in $D^{\prime}$. Let $S^{\prime}:=\left(S-\left\{y_{1}, y_{2}\right\}\right) \cup\{x\}$. Since $d_{D^{\prime}}^{-}(x)=d_{D^{\prime}}^{+}\left(y_{1}\right)=d_{D^{\prime}}^{+}\left(y_{2}\right)=0$, we see that $|(S, \bar{S})|=\left|\left(S^{\prime}, \overline{S^{\prime}}\right)\right|$. So $\left(S^{\prime}, \overline{S^{\prime}}\right)$ gives the desired maximum dicut in $D^{\prime}$.

Suppose $\gamma\left(D^{\prime}\right) \geq 2 / 5$. Then $D^{\prime}$ has a dicut of size at least $(2 / 5) \varepsilon\left(D^{\prime}\right)$. By $(a)$, such a dicut in $D^{\prime}$, say $(S, \bar{S})$, may be chosen so that $x \in S$ and $\left\{y_{1}, y_{2}\right\} \subseteq \bar{S}$. Now $(S, \bar{S})$, when viewed as a dicut in $D$, also contains $\left(x, y_{1}\right)$ and $\left(x, y_{2}\right)$; and hence has at least $(2 / 5) \varepsilon\left(D^{\prime}\right)+2 \geq(2 / 5) \varepsilon(D)$ edges (since $\left.\varepsilon\left(D^{\prime}\right) \geq \varepsilon(D)-5\right)$.

Given a digraph $D$, we let $V_{1}(D)=\left\{u \in V(D): d_{D}^{+}(u) \geq 2\right\}$ and $V_{2}(D)=\{u \in V(D)$ : $\left.d_{D}^{-}(u) \geq 2\right\}$. Further, let $B_{i}(D)$ denote the subgraph of $D$ induced by $V_{i}(D)$. When no confusion arises, we simply use $V_{i}$ and $B_{i}$ instead of $V_{i}(D)$ and $B_{i}(D)$. 
Lemma 2.2 Let $D \in D(1,1)$, and assume that $D$ is irreducible and $V_{1} \cup V_{2} \neq \emptyset$. Then

(a) each nonempty $B_{i}$ consists of vertex disjoint directed cycles,

(b) $\left(\overline{V_{1}}, V_{1}\right)=\emptyset=\left(V_{2}, \overline{V_{2}}\right)$,

(c) each vertex in $V_{1}$ (respectively, $\left.V_{2}\right)$ is incident with exactly one edge in $\left(V_{1}, \overline{V_{1}}\right)$ (respectively, $\left.\left(\overline{V_{2}}, V_{2}\right)\right)$,

(d) the vertices in $V_{1} \cup V_{2}$ are precisely the 3 -vertices of $D$, and

(e) $D$ is subcubic, and if $D \in \mathcal{D}$ then $D$ is triangle-free.

Proof. Since $D \in D(1,1), d_{D}^{-}(x) \leq 1$ for all $x \in V_{1}$, and $d_{D}^{+}(y) \leq 1$ for all $y \in \overline{V_{1}}$. Since $D$ is irreducible, every vertex in $V_{1}$ is incident with at most one edge in $\left(V_{1}, \overline{V_{1}}\right)$. Then $d_{B_{1}}^{+}(x) \geq 1$ for all $x \in V_{1}$. By definition, $d_{B_{1}}^{-}(x) \leq d_{D}^{-}(x) \leq 1$ for all $x \in V_{1}$. Hence

$$
\left|V_{1}\right| \leq \sum_{x \in V_{1}} d_{B_{1}}^{+}(x)=\sum_{x \in V_{1}} d_{B_{1}}^{-}(x) \leq\left|V_{1}\right| .
$$

This implies that $d_{B_{1}}^{+}(x)=d_{B_{1}}^{-}(x)=d_{D}^{-}(x)=1$ for all $x \in V_{1}$. So (a)-(c) hold for $V_{1}$, and the vertices of $V_{1}$ are 3 -vertices of $D$.

By applying the same argument to $D^{r}$ and noting that $V_{1}\left(D^{r}\right)=V_{2}(D)$, we can show that (a)-(c) also hold for $V_{2}$, and the vertices of $V_{2}$ are 3-vertices of $D$.

Clearly, $V_{2} \subseteq \overline{V_{1}}$ (since $D \in D(1,1)$ ), and every vertex $y \in \overline{V_{1}}-V_{2}$ has degree at most 2 (since $d_{D}^{+}(y) \leq 1$ and $\left.d_{D}^{-}(y) \leq 1\right)$. So $(\mathrm{d})$ holds, and $D$ is subcubic.

Now assume $D \in \mathcal{D}$. It remains to show that $D$ is triangle-free. Let $B$ denote the subgraph of $D$ induced by $\overline{V_{1}}-V_{2}$. Recall that for all $y \in V(B)$, we have $d_{D}^{-}(y) \leq 1 \geq d_{D}^{+}(y)$. So if $B$ contains a cycle then it must be a directed cycle and is a component of $D$, which is impossible since $D$ is connected and $V_{1} \cup V_{2} \neq \emptyset$. Hence $B$ contains no cycles.

Now suppose that $D$ contains a triangle, say $T$. Then, $T \nsubseteq B$ because $B$ has no cycles. Since $D \in \mathcal{D}, T$ is not a directed triangle. So by (a), $T \nsubseteq B_{i}$ for $i \in\{1,2\}$.

If $\left|V(T) \cap V_{1}\right|=1$ (or $\left|V(T) \cap V_{2}\right|=1$ ) then by (b), the vertex in $V(T) \cap V_{1}$ (or $\left.V(T) \cap V_{2}\right)$ is incident with two edges in $\left(V_{1}, \overline{V_{1}}\right)$ (or $\left(\overline{V_{2}}, V_{2}\right)$ ), contradicting (c). If $\left|V(T) \cap V_{1}\right|=2$ and $|V(T) \cap V(B)|=1$ then by (b), the vertex in $V(T) \cap V(B)$ has indegree at least 2, a contradiction. So $\left|V(T) \cap V_{2}\right|=2$ and $|V(T) \cap V(B)|=1$. Then by (b), the vertex in $V(T) \cap V(B)$ has outdegree at least 2 , a contradiction.

We need a result of Bondy and Locke [4] on max cuts in triangle-free subcubic graphs.

Theorem 2.3 (Bondy and Locke [4]) If $G$ is a triangle-free subcubic graph, then $G$ has a cut of size at least $(4 / 5) \varepsilon(G)$, and such a cut can be found in $O\left(|V(G)|^{2}\right)$ time.

We now give an alternative proof of the result of Lehel et al.

Theorem 2.4 (Lehel et al. [13]) If $D \in \mathcal{D}$ then $\gamma(D) \geq 2 / 5$. 
Proof. It is straightforward to check that the assertion of the theorem holds when $\varepsilon(D) \leq 5$, as well as when $D$ is a directed cycle or directed path. So we may assume that $\varepsilon(D) \geq 6$ and $V_{1} \cup V_{2} \neq \emptyset$, and that the assertion of the theorem holds for digraphs in $\mathcal{D}$ with size less than $\varepsilon(D)$.

If there exist $x \in V_{1}$ and distinct $y_{1}, y_{2} \in \overline{V_{1}}$ such that $\left\{\left(x, y_{1}\right),\left(x, y_{2}\right)\right\} \subseteq E(D)$, then every nontrivial component of $D^{\prime}:=R\left(D, x, y_{1}, y_{2}\right)$ belongs to $\mathcal{D}$. By applying induction to each nontrivial component of $D^{\prime}$, we can show that $\gamma\left(D^{\prime}\right) \geq 2 / 5$. It follows from Lemma 2.1(b) that $\gamma(D) \geq 2 / 5$.

So we may assume $D$ is not reducible. By applying the same argument to $D^{r}$, we may also assume that $D^{r}$ is not reducible. Therefore, $D$ is irreducible. So by Lemma 2.2(e), $D$ is subcubic and triangle-free. By Theorem 2.3, the underlying graph of $D$ has a cut of size at least $(4 / 5) \varepsilon(D)$. Hence $\gamma(D) \geq 2 / 5$.

Note that the lower bound in Theorem 2.4 is attained by the digraph $A$ in Figure 5 and by the directed pentagon. The proof of Theorem 2.4 gives the following algorithm which, given a digraph from $\mathcal{D}$, finds a dicut with at least $(2 / 5) \varepsilon(D)$ edges.

\section{Algorithm DICUT}

Input A digraph $D$ whose components all belong to $\mathcal{D}$.

Output A dicut $(S, \bar{S})$ of size at least $(2 / 5) \varepsilon(D)$.

Set $i \leftarrow 0$ and $D_{0} \leftarrow D$

1. Find the sets $V_{1}\left(D_{i}\right)$ and $V_{2}\left(D_{i}\right)$.

2. If there exist $x \in V_{1}\left(D_{i}\right)$ and distinct $y_{1}, y_{2} \in \overline{V_{1}\left(D_{i}\right)}$ such that $\left\{\left(x, y_{1}\right),\left(x, y_{2}\right)\right\} \subseteq E\left(D_{i}\right)$, then let $D_{i+1}:=R\left(D_{i}, x, y_{1}, y_{2}\right)$. Set $i \leftarrow i+1$ and $D_{i} \leftarrow D_{i+1}$, and go to step 1 .

3. If there exist $x \in V_{2}\left(D_{i}\right)$ and distinct $y_{1}, y_{2} \in \overline{V_{2}\left(D_{i}\right)}$ such that $\left\{\left(y_{1}, x\right),\left(y_{2}, x\right)\right\} \subseteq E\left(D_{i}\right)$, then let $D_{i+1}:=\left(R\left(D_{i}^{r}, x, y_{1}, y_{2}\right)\right)^{r}$. Set $i \leftarrow i+1$ and $D_{i} \leftarrow D_{i+1}$, and go to step 1 .

4. Suppose $D_{i}$ is irreducible. If $V_{1}\left(D_{i}\right) \cup V_{2}\left(D_{i}\right)=\emptyset$ then every component of $D_{i}$ is a directed path or directed cycle, and we can find a dicut $\left(S_{i}, \overline{S_{i}}\right)$ in $D_{i}$ with at least $(2 / 5) \varepsilon\left(D_{i}\right)$ edges. If $V_{1}\left(D_{i}\right) \cup V_{2}\left(D_{i}\right) \neq \emptyset$ then $D_{i}$ is subcubic and triangle-free. Apply the Bondy-Locke algorithm in Theorem 2.3 to find a max cut in the underlying graph of $D_{i}$ with at least $(4 / 5) \varepsilon\left(D_{i}\right)$ edges. Then find a dicut $\left(S_{i}, \overline{S_{i}}\right)$ in $D_{i}$ with at least $(2 / 5) \varepsilon\left(D_{i}\right)$ edges.

5. Set $j \leftarrow i$.

6. If $D_{j}=D$ then set $S \leftarrow S_{j}$ and output $(S, \bar{S})$; otherwise go to step 7 .

7. If $D_{j}$ is obtained from $D_{j-1}$ as in step 2 then modify $\left(S_{j}, \overline{S_{j}}\right)$ so that $x \in S_{j}$ and $\left\{y_{1}, y_{2}\right\} \subseteq$ $\overline{S_{j}}$; if $D_{j}$ is obtained from $D_{j-1}$ as in step 3 , then modify $\left(S_{j}, \overline{S_{j}}\right)$ so that $x \in \overline{S_{j}}$ and $\left\{y_{1}, y_{2}\right\} \subseteq S_{j}$. Set $j \leftarrow j-1$, and $D_{j} \leftarrow D_{j-1}$, and go to step 6 .

Note that steps 1, 2 and 3 each execute $O(|E(D)|)$ time. Step 4 requires $O\left(|V(D)|^{2}\right)$. Steps 5,6 and 7 each require constant time. So it is not difficult to see that the running time of the above algorithm is $O\left(|V(D)|^{3}\right)$.

We now construct a sequence of subcubic digraphs $X_{0}, X_{1}, \ldots, X_{i} \ldots$, such that for each $i \geq 0$, $X_{i}$ contains exactly $i+1$ directed triangles and $\gamma\left(X_{i}\right)=(1+2 i) /(3+5 i)<2 / 5$. Let $X$ denote the 


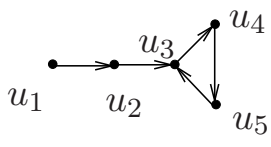

$X$

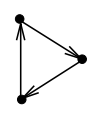

$X_{0}$

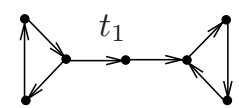

$X_{1}$

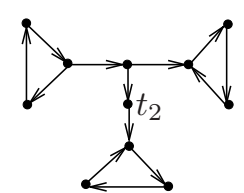

$X_{2}$

Figure 1: Graphs $X, X_{0}, X_{1}$ and $X_{2}$

digraph with $V(X)=\left\{u_{1}, u_{2}, u_{3}, u_{4}, u_{5}\right\}$ and $E(X)=\left\{\left(u_{1}, u_{2}\right),\left(u_{2}, u_{3}\right),\left(u_{3}, u_{4}\right),\left(u_{4}, u_{5}\right),\left(u_{5}, u_{3}\right)\right\}$. See Figure 1.

For convenience, let $X_{0}$ denote the directed triangle. We define $X_{i}, i \geq 1$, in the following recursive fashion. Let $X_{1}$ be the digraph obtained from $X_{0}$ and $X$ by identifying $u_{1}$ with a vertex of $X_{0}$. (Note that $u_{2}$ is the unique 2-vertex of $X$ (also of $X_{1}$ ) that is not on any triangle.) For $i \geq 1, X_{i}$ has a unique 2 -vertex that is not on any triangle, and we denote it by $t_{i}$. Let $X_{i+1}$ be the digraph obtained from $X_{i}$ and $X$ by identifying $t_{i}$ with $u_{1} . X_{1}$ and $X_{2}$ are given in Figure 1 .

By definition, $\varepsilon\left(X_{i}\right)=3+5 i$. To show that $\gamma\left(X_{i}\right)=(1+2 i) /(3+5 i)$, it suffices to prove that every maximum dicut of $X_{i}$ has exactly $1+2 i$ edges. This is certainly true when $i=0$. Now assume for some $k \geq 1$, every maximum dicut of $X_{k-1}$ has $1+2(k-1)$ edges.

Since every maximum dicut of $X$ has two edges, if $X_{k}$ has a dicut of size $l$ then $X_{k-1}$ has a dicut of size at least $l-2$. Hence, a maximum dicut of $X_{k}$ has at most $1+2(k-1)+2=1+2 k$ edges.

Let $x^{\prime}$ be the vertex of $X_{k}$ which is the result of the identification of $t_{k-1}$ and $u_{1}$. Let $(S, \bar{S})$ be a maximum dicut of $X_{k-1}$. If $t_{k-1} \in S$ then let $S^{\prime}=\left(S-\left\{t_{k-1}\right\}\right) \cup\left\{x^{\prime}, u_{4}\right\}$; and otherwise let $S^{\prime}=S \cup\left\{u_{2}, u_{4}\right\}$. Then, $\left(S^{\prime}, \overline{S^{\prime}}\right)$ is a dicut of $X_{k}$ and has $2 k+1$ edges.

Therefore, we have shown that any maximum dicut of $X_{k}$ has precisely $1+2 k$ edges, and so, $\gamma\left(X_{k}\right)=(1+2 k) /(3+5 k)$.

\section{$3 \quad$ Extremal graphs}

By Theorem 2.4, every digraph in $\mathcal{D}$ has dicut density at least $2 / 5$. In this section, we investigate the extremal graphs. First, we show that when the operation introduced in the previous section is applied to an extremal graph, the resulting graph (when nontrivial) is also extremal.

Lemma 3.1 Let $D \in \mathcal{D}$ and $x \in V(D)$, let $\left(x, y_{i}\right) \in E(D)$ with $d_{D}^{+}\left(y_{i}\right) \leq 1$ for $i \in\{1,2\}$, and let $D^{\prime}:=R\left(D, x, y_{1}, y_{2}\right)$. If $\gamma(D)=2 / 5$ and $\varepsilon(D) \geq 10$, then $\gamma\left(D^{\prime}\right)=2 / 5$ and $d_{D}^{-}(x)=d_{D}^{+}\left(y_{1}\right)=$ $d_{D}^{+}\left(y_{2}\right)=1$.

Proof. Suppose $\gamma(D)=2 / 5$ and $\varepsilon(D) \geq 10$. Then $\varepsilon(D)=5 k$ for some integer $k \geq 2$. Since $D \in \mathcal{D}$ and $d_{D}^{+}(x) \geq 2$, we have $d_{D}^{-}(x) \leq 1$. So $\varepsilon\left(D^{\prime}\right) \geq \varepsilon(D)-5=5 k-5 \geq 5$ and each nontrivial component of $D^{\prime}$ belongs to $\mathcal{D}$. By applying Theorem 2.4 to the nontrivial components of $D$, we can show that $\gamma\left(D^{\prime}\right) \geq 2 / 5$.

Assume $\gamma\left(D^{\prime}\right)>2 / 5$, and let $(S, \bar{S})$ be a maximum dicut of $D^{\prime}$. Then, $|(S, \bar{S})|>(2 / 5) \varepsilon\left(D^{\prime}\right) \geq$ $(2 / 5)(5 k-5)=2 k-2$. Hence $|(S, \bar{S})| \geq 2 k-1$. By Lemma 2.1, we may assume $x \in S$ and 
$y_{1}, y_{2} \in \bar{S}$. Hence $(S, \bar{S})$, when viewed as a dicut in $D$, also contains $\left(x, y_{1}\right)$ and $\left(x, y_{2}\right)$; and thus has size at least $(2 k-1)+2=2 k+1$, contradicting the assumption that $\gamma(D)=2 / 5$.

Therefore, $\gamma\left(D^{\prime}\right)=2 / 5$. So $\varepsilon\left(D^{\prime}\right)$ is a multiple of 5 ; hence $d_{D}^{-}(x)=d_{D}^{+}(y)=d_{D}^{+}(z)=1$.

However, if $\gamma(D)>2 / 5$ then applying our operation to $D$ or $D^{r}$ may result in a digraph with dicut density 2/5. For example, let $Y$ denote the digraph with vertex set $\left\{x_{0}, x_{1}, \ldots, x_{5}\right\}$ and edge set $\left\{\left(x_{0}, x_{1}\right),\left(x_{1}, x_{2}\right),\left(x_{2}, x_{3}\right),\left(x_{1}, x_{4}\right),\left(x_{4}, x_{5}\right)\right\}$. See Figure 2 . The digraphs $D_{1}$ and $D_{2}$ are obtained by identifying the 1-vertices of $Y$ with certain vertices of the directed pentagon. (The edges of $Y$ are thickened.) In each $D_{i}$, the edges from squares to circles form a dicut, showing that $\gamma\left(D_{i}\right) \geq 1 / 2>2 / 5$.
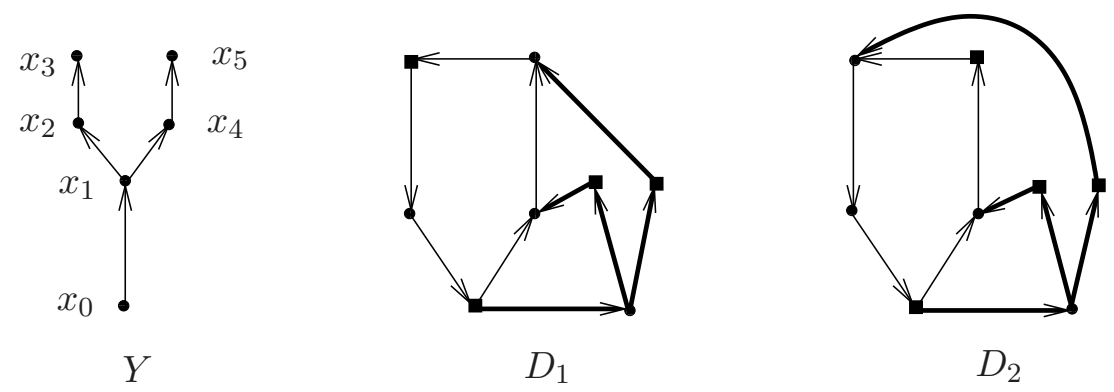

Figure 2: Adding $Y$ to the directed pentagon

To characterize the irreducible digraphs in $\mathcal{D}$ that have dicut density $2 / 5$, we also need the following result which was conjectured by Bondy and Locke [4], and proved recently by the present authors [16].

Theorem 3.2 ( $X u$ and $Y u$ [16]) If the graph $G$ is triangle-free and subcubic, and if each maximum cut of $G$ has exactly $(4 / 5) \varepsilon(G)$ edges, then $G$ is one of the graphs in Figure 3.
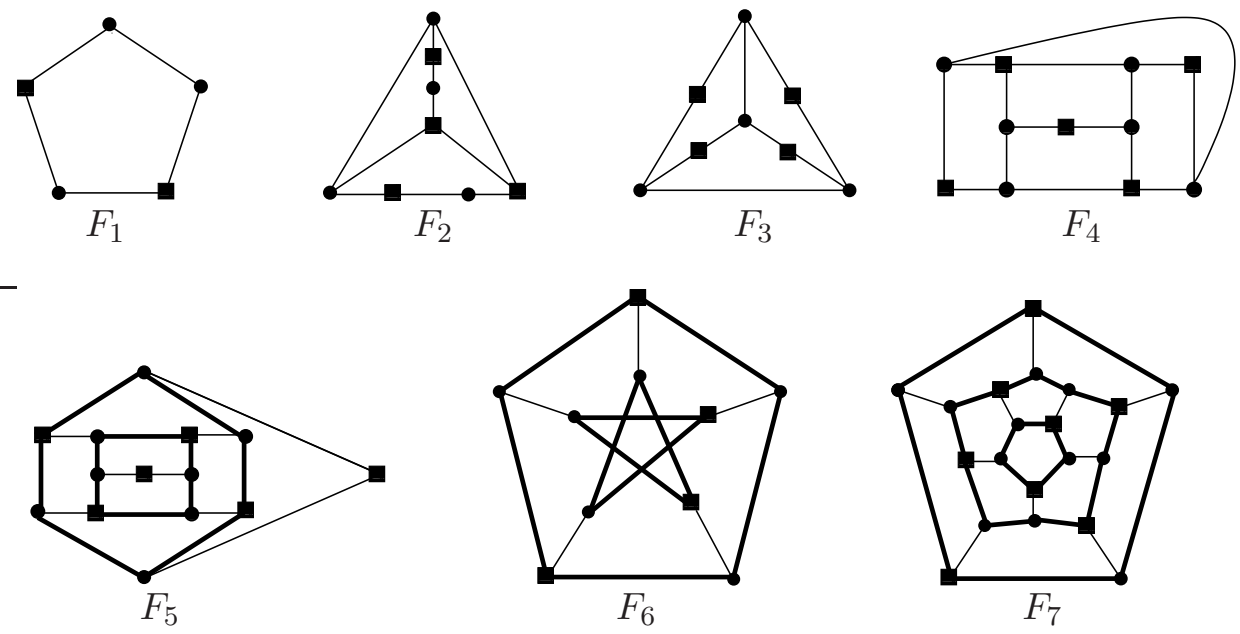

Figure 3: Extremal graphs for Theorem 2.3. 
Note that $F_{6}$ is the Petersen graph and $F_{7}$ is the dodecahedron. For each $F_{i}$ in Figure 3 , the edges between squares and circles form a maximum cut of size $(4 / 5) \varepsilon\left(F_{i}\right)$.

Also note that $F_{6}$ consists of two disjoint pentagons $C_{1}, C_{2}$ (thickened) and a matching between $C_{1}$ and $C_{2}$. Let $P$ denote the orientation of $F_{6}$ so that $C_{1}$ and $C_{2}$ are directed cycles, and the matching edges are oriented from $C_{1}$ to $C_{2}$. Note that such an orientation of $F_{6}$ is unique up to isomorphism. Clearly, $P=P^{r}$ and $P$ is irreducible.

Proposition 3.3 Let $P$ be the digraph defined above. Then $\gamma(P)=2 / 5$.

Proof. Let $V\left(C_{1}\right)=\left\{v_{i}: 1 \leq i \leq 5\right\}$ and $E\left(C_{1}\right)=\left\{\left(v_{i}, v_{i+1}\right): 1 \leq i \leq 5\right\}$ where $v_{6}=v_{1}$, and let $V\left(C_{2}\right)=\left\{u_{i}: 1 \leq i \leq 5\right\}$ such that $\left(v_{i}, u_{i}\right) \in E(P)$ for $1 \leq i \leq 5$. Let $(S, \bar{S})$ be a maximum dicut in $P$, and for $i=1,2$, let $S_{i} \subseteq S$ consist of the vertices that are incident with $i$ edges in $(S, \bar{S})$. Then $S_{2} \subseteq V\left(C_{1}\right)$, and $S_{2}$ (if nonempty) is an independent set in $P$. Hence $\left|S_{2}\right| \leq 2$.

Since $|(S, \bar{S})| \geq 6$ (by Theorem 3.2), it suffices to show that $|(S, \bar{S})| \leq 6$. Assume to the contrary that $|(S, \bar{S})| \geq 7$. Then $\left|S_{1}\right| \geq 7-2\left|S_{2}\right|$.

If $\left|S_{2}\right|=0$, then $\left|S_{1}\right| \geq 7$, and hence $|\bar{S}| \leq 3$. However, this implies (by pigeon-hole principle) that there is some $v \in \bar{S}$ such that $d^{-}(v) \geq 3$, a contradiction.

If $\left|S_{2}\right|=2$, we may assume (by symmetry) $S_{2}=\left\{v_{1}, v_{3}\right\}$. Then $\left\{u_{1}, u_{3}, v_{2}, v_{4}\right\} \subseteq \bar{S},\left\{u_{5}, v_{5}\right\} \nsubseteq$ $S_{1}$, and $\left\{u_{2}, u_{4}\right\} \nsubseteq S_{1}$. This forces $\left|S_{1}\right| \leq 2$, a contradiction since $\left|S_{1}\right| \geq 7-2\left|S_{2}\right|=3$.

Assume $\left|S_{2}\right|=1$, and assume without loss of generality that $S_{2}=\left\{v_{1}\right\}$. Then $\left\{u_{1}, v_{2}\right\} \subseteq \bar{S}$ and $\left|S_{1}\right| \geq 5$. Since $\left|\left\{u_{2}, \ldots, u_{5}\right\} \cap S_{1}\right| \leq 2$ and $\left|S_{1}\right| \geq 5,\left\{v_{3}, v_{4}, v_{5}\right\} \subseteq S_{1}$. But this forces $\left\{u_{3}, u_{4}, u_{5}\right\} \subseteq \bar{S}$, and thus $\left|S_{1}\right| \leq 4$, a contradiction.

Theorem 3.4 Let $D \in \mathcal{D}$ be irreducible. If $\gamma(D)=2 / 5$, then $D=P$ or $D$ is the directed pentagon.

Proof. Assume $\gamma(D)=2 / 5$. Let $G$ be the underlying graph of $D$. Recall Lemma 2.2 and related notation. If $V_{1} \cup V_{2}=\emptyset$ then $G$ is a path or cycle, which implies that $D$ is the directed pentagon (since $\gamma(D)=2 / 5$ ). So we may assume $V_{1} \cup V_{2} \neq \emptyset$. Then by Lemma $2.2(\mathrm{e}), G$ is subcubic and triangle-free. By Theorem 3, every maximum cut of $G$ has size at least $(4 / 5) \varepsilon(G)$. Since $\gamma(D)=2 / 5$, every maximum cut of $G$ has size precisely $(4 / 5) \varepsilon(G)$. So by Theorem $3.2, G$ is one of the graphs in Figure 3. Since $V_{1} \cup V_{2} \neq \emptyset, G \neq F_{1}$.

By symmetry between $D$ and $D^{r}$, we may assume $V_{1} \neq \emptyset$. By Lemma $2.2(\mathrm{c}), V_{1} \neq V(D)$, and so $V_{2} \neq \emptyset$. Hence by Lemma 2.2(a), each $B_{i}$ consists of disjoint directed cycles. So by Lemma 2.2(d), $G \notin\left\{F_{2}, F_{3}, F_{4}\right\}$; and by Lemma $2.2(\mathrm{~b})$ and (c), $D=P$ when $G=F_{6}$. In view of Propositions 3.3, it remains to show that $G \notin\left\{F_{5}, F_{7}\right\}$.

Suppose $G=F_{5}$. Note that the 3-vertices of $G$ induce two disjoint hexagons (which are thickened in Figure 3). By Lemma 2.2(a), each nonempty $B_{i}$ consists of disjoint directed hexagons. Let $x$ be an arbitrary 2-vertex of $G$. Then $d_{D}^{+}(x)=1=d_{D}^{-}(x)$. Since both neighbors of $x$ are contained in the same directed hexagon, both neighbors of $x$ must be contained in the same $B_{i}$. This implies that either $\left(\overline{V_{1}}, V_{1}\right) \neq \emptyset$ or $\left(V_{2}, \overline{V_{2}}\right) \neq \emptyset$, contradicting Lemma $2.2(\mathrm{~b})$. So $G \neq F_{5}$.

Now assume $G=F_{7}$. Note that the dodecahedron consists of two disjoint induced pentagons $C_{1}$ and $C_{3}$, an induced cycle $C_{2}$ of length 10, and a perfect matching from $C_{1} \cup C_{3}$ to $C_{2}$. See $F_{7}$ in Figure 3, where $C_{1}, C_{2}$ and $C_{3}$ are thickened. Note that, up to isomorphism, such a partition of $F_{7}$ is unique. By Lemma 2.2(a), $C_{1}, C_{2}, C_{3}$ are all directed cycles in $D$. Since $\gamma(D)=2 / 5, V_{1}$ and $V_{2}$ must be $V\left(C_{1} \cup C_{3}\right)$ and $V\left(C_{2}\right)$. By Lemma 2.2(b), either all matching edges are oriented from $C_{2}$ to $C_{1} \cup C_{3}$, or all matching edges are oriented from $C_{1} \cup C_{3}$ to $C_{2}$. By a simple case 
analysis, we see that $F_{7}$ has only six nonisomorphic orientations: three are shown in Figure 4, and the other three can be obtained by reversing the orientations of all edges in those shown in Figure 4. In each of these orientations, the edges between squares and circles form a dicut of size 13. This contradicts the assumption that $\gamma(D)=2 / 5$. So $G \neq F_{7}$.
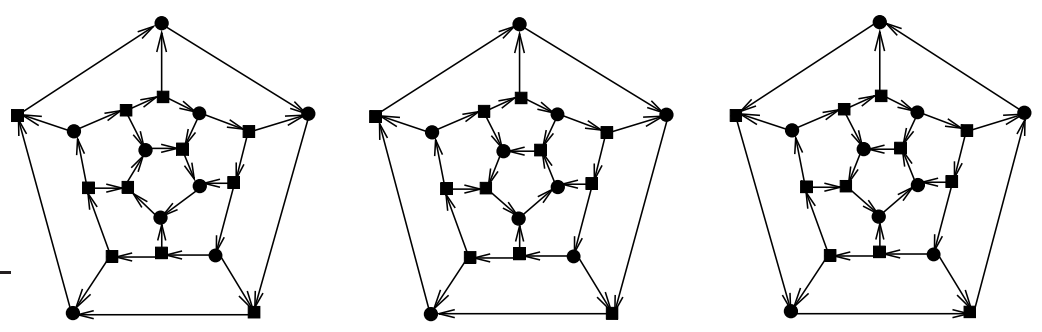

Figure 4: Three orientations of $F_{7}$ with dicuts of size 13.

Note that the digraph $A$ in Figure 5 is reducible. We now characterize all acyclic digraphs in $D(1,1)$ with dicut density $2 / 5$. We show that such a graph must contain $A$ as a subgraph, and can be obtained by gluing copies of $A$ appropriately.

To be precise, we define an ordering on the vertices of any acyclic digraph as follows: $x<y$ iff there is a directed path from $x$ to $y$ (this is a partial ordering because the digraph is acyclic). Given two acyclic digraphs $D_{1}$ and $D_{2}$, we can produce an acyclic digraph from $D_{1}, D_{2}$ as follows: Take vertices $x_{1}<x_{2}<\ldots<x_{m}$ of $D_{1}$ and $y_{1}<y_{2}<\ldots<y_{m}$ of $D_{2}$, and identify $x_{i}$ with $y_{i}$ for $1 \leq i \leq m$. We say that the resulting digraph is obtained by gluing $D_{1}$ and $D_{2}$.

Let $\mathcal{T}$ denote the class of digraphs in $D(1,1)$ with at least one nontrivial component such that every nontrivial component of each member of $\mathcal{T}$ can be obtained by gluing a finite number of copies of $A$. Figure 5 shows several digraphs in $\mathcal{T}$. The second digraph is obtained by identifying the 1-vertices of two copies of $A$, the third is obtained by identifying a 1-vertex of $A$ with a 1-vertex of another copy of $A$, and the fourth is obtained by identifying a 1-vertex of $A$ with a 2-vertex of another copy of $A$.
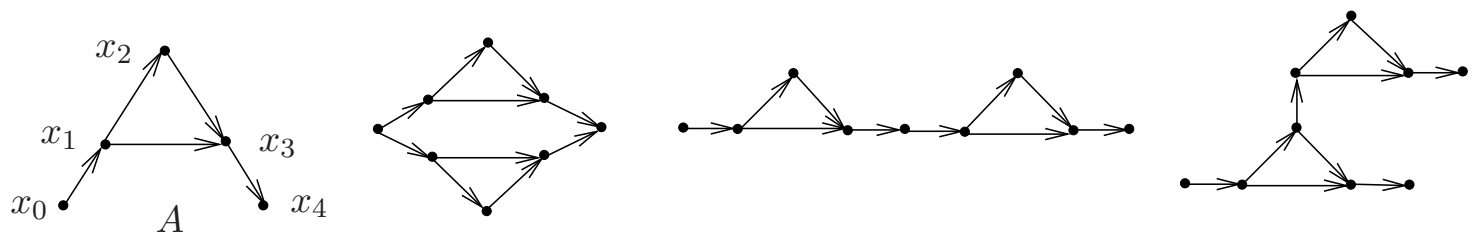

Figure 5: Directed graph $A$ and three digraphs in $\mathcal{T}$

Since $A^{r}=A, T \in \mathcal{T}$ iff $T^{r} \in \mathcal{T}$. We now show that every member of $\mathcal{T}$ has dicut density $2 / 5$. Let $T \in \mathcal{T}$. Without loss of generality, we may assume $T$ is connected. By Theorem 2.4, $\gamma(T) \geq 2 / 5$. By the definition of $\mathcal{T}, \varepsilon(T)=5 k$ for some integer $k \geq 1$. If $k=1$ then $T=A$ and hence $\gamma(T)=2 / 5$. So we may assume that $k \geq 2$, and $\gamma(T)=2 / 5$ when $5 \leq \varepsilon(T)<5 k$. Let $T$ be obtained by gluing $A$ and some $T^{\prime} \in \mathcal{T}$. Since every dicut of $T$ contains at most two edges from $A$, any maximum dicut of $T^{\prime}$ contains at least $5 k \gamma(T)-2$ edges. If $\gamma(T)>2 / 5$, then $5 k \gamma(T)-2>2 k-2$. That means $T^{\prime}$ contains a dicut of size at least $2 k-1$, a contradiction. 
Theorem $3.5 \mathcal{T}$ consists of precisely those acyclic digraphs in $D(1,1)$ with dicut density $2 / 5$.

Proof. Since $\mathcal{T} \subseteq D(1,1)$ and $\gamma(T)=2 / 5$ for all $T \in \mathcal{T}$, it suffices to show that every connected acyclic digraph in $D(1,1)$ with dicut density $2 / 5$ belongs to $\mathcal{T}$. Let $D \in D(1,1)$ be a connected acyclic digraph with $\gamma(D)=2 / 5$. Then $\varepsilon(D)=5 k$ for some integer $k \geq 1$. If $k=1$ then $D=A \in \mathcal{T}$. So we may assume $k \geq 2$ and that $D^{\prime} \in \mathcal{T}$ for all connected acyclic digraphs $D^{\prime} \in D(1,1)$ with $\gamma\left(D^{\prime}\right)=2 / 5$ and $\varepsilon\left(D^{\prime}\right) \leq 5(k-1)$.

We now prove $A \subseteq D$. By applying Lemma 3.1 repeatedly (starting with $D$, then the nontrivial components of the resulting graph, and so on), we arrive at a digraph $H$ whose components either are trivial or irreducible, or have precisely 5 edges; and not all components are trivial. Let $K$ be any nontrivial component of $H$. Then by Lemma 3.1, $\gamma(K)=2 / 5$. If $K$ is irreducible then by Theorem 3.4, $K=P$ or $K$ is the directed pentagon, a contradiction since $D$ is acyclic. So $K$ has precisely 5 edges. Since $K$ is acyclic and $\gamma(K)=2 / 5$, we have $K=A$.

Let the vertices of $A$ be labeled as in Figure 5 . If $\left(x_{2}, x_{3}\right)$ is the unique edge leaving $x_{2}$, then by Lemma 3.1, $H=D-E(A)$ is an acyclic digraph in $D(1,1)$ with $\varepsilon(H)=5(k-1)$ and $\gamma(H)=2 / 5$. By our induction hypothesis, $H \in \mathcal{T}$. Hence $D$ is obtained by gluing $A$ and $H$, which shows $D \in \mathcal{T}$.

If $d_{D}^{+}\left(x_{2}\right) \geq 2$, then $\left(x_{1}, x_{2}\right)$ is the unique edge ending at $x_{2}$. In $D^{r},\left(x_{1}, x_{0}\right)$ is the unique edge leaving $x_{1},\left(x_{2}, x_{1}\right)$ is the unique edge leaving $x_{2}$. By applying the same argument as above to $D^{r}$ and $A^{r}=A$, we can show that $D^{r} \in \mathcal{T}$. Hence $D \in \mathcal{T}$.

As a consequence, we have an $O(|E(D)|)$ algorithm which decides whether an acyclic digraph $D$ in $D(1,1)$ has dicut density $2 / 5$.

\section{Algorithm ACYCLIC}

Input: Acyclic digraph $D \in D(1,1)$.

Output: YES, if $\gamma(D)=2 / 5$; NO, if $\gamma(D)>2 / 5$.

1. Search in $D$ for maximal sequence of edge disjoint copies $A_{1}, \ldots, A_{k}$ of $A$. If $k=0$ then stop and output NO. Otherwise, let the vertices of each $A_{i}$ be labeled as in Figure 5, and go to step 2 .

2. Set $D_{0} \leftarrow D$. For each $1 \leq i \leq k$, if $d_{D_{i-1}}^{+}\left(x_{2}\right)=1$ then let $D_{i}:=R\left(D_{i-1}, x_{1}, x_{2}, x_{3}\right)$, and if $d_{D_{i-1}}^{+}\left(x_{2}\right) \geq 2$ then let $D_{i}:=\left(R\left(D_{i-1}^{r}, x_{3}, x_{1}, x_{2}\right)\right)^{r}$.

3. If $D_{k}=\emptyset$ then $D \in \mathcal{T}$ and output YES. Otherwise $D \notin \mathcal{T}$ and output NO.

Clearly, step 1 runs in $O(|E(D)|)$ time, and steps 2 and 3 each run in constant time. So the running time of Algorithm ACYCLIC is $O(|E(D)|)$. 


\section{References}

[1] N. Alon, Bipartite subgraphs, Combinatorica 16 (1996) 301-311.

[2] N. Alon, B. Bollobás, A. Gyárfás, J. Lehel and A. Scott, Maximum dicuts in acyclic digraphs, J. Graph Theory 55 (2007) 1-13.

[3] P. Berman and M. Karpinski, On some tighter inapproximability results, (extended abstract) Lecture Notes in Computer Science Springer, Berlin, Heidelberg, Volume 1644 (1999) 200209.

[4] J. A. Bondy and S. C. Locke, Largest bipartite subgraphs in triangle-free graphs with maximum degree three, J. Graph Theory 10 (1986) 477-504.

[5] M. Cropper, A. Gyárfás and J. Lehel, Hall ratio of the Mycielski graphs, Discrete Math. 306 (2006) 1988-1990.

[6] A. Daneshgar, A. J. W. Hilton and P. D. Johnson Jr., Relations among the fractional chromatic, choice, Hall, and Hall-condition numbers of simple graphs, Discrete Math. 241 (2001) 189-199.

[7] C. S. Edwards, Some extremal properties of bipartite graphs, Canadian J. Math. 25 (1973) 475-485.

[8] U. Feige, M. Karpinski, and M. Langberg, Improved approximation of max-cut on graphs of bounded degree, J. Algorithms 43 (2002) 201-219.

[9] M. X. Goemans and D. P. Williamson, Improved approximation algorithms for maximum cut and satisfiability using semidefinite programming, J. ACM 42 (1995) 1115-1145.

[10] G. Hopkins and W. Staton, Extremal bipartite subgraphs of cubic triangle-free graphs, $J$. Graph Theory 6 (1982) 115-121.

[11] P. D. Johnson, Jr., The hall condition number of a graph, Ars Combinatoria 37 (1994) 183-190.

[12] R. M. Karp, Reducibility among combinatorial problems, in Complexity of Computer Computations (R. Miller and J. Thatcher, eds.) Plenum Press, New York (1972) 85-103.

[13] J. Lehel, F. Maffray and M. Preissmann, Maximum directed cuts in digraphs with degree restriction, J. Graph Theory, to appear.

[14] S. C. Locke, Maximum k-colourable subgraphs, J. Graph Theory 6 (1982) 123-132.

[15] W. Staton, Edge deletions and the chromatic number, Ars Combinatoria 10 (1980) 103-106.

[16] B. Xu and X. Yu, Triangle-free subcubic graphs with minimum bipartite density, J. Combin. Theory Ser. B 98 (2008) 516-537.

[17] M. Yannakakis, Node- and Edge-Deletion NP-Complete Problems, STOC (1978) 253-264. 